\title{
Minimum Standards for Clinical Evoked Potential Studies
}

\section{Canadian Society of Clinical Neurophysiologists}

Evoked potentials are the electrical changes that occur in the brain in response to sensory stimuli. They can be recorded from scalp electrodes using sensitive amplifiers. Averaging techniques are necessary to separate the evoked potentials from other electrical activities. Evoked potentials can provide helpful information for the clinical evaluation of neurological patients. ${ }^{1,2}$ They can assess sensory function by demonstrating the brain's response to sensory stimuli. They can localize dysfunction in sensory pathways by showing where and when a sensory response becomes abnormal. They can identify abnormalities of sensory processing that are not apparent during the usual clinical examination. They can assess the extent of normal or abnormal function and thereby assist prognosis in patients with brain-damage.

Several standards for evoked potential studies have been published. ${ }^{3-6}$ The standards described in this report were put together under the auspices of the Canadian Society of Clinical Neurophysiologists and accepted at the society's annual meeting in Toronto on June 18, 1993. The standards derive from those previously published with some adjustment to fit the context of Canadian medicine. They are designed basically for evoked potential studies of neurological or neurosurgical patients. They do not cover the uses of sensory evoked potentials in other fields such as the evaluation of hearing thresholds in audiology or the study of visual function in ophthalmology. These standards describe the minimum necessary conditions for recording and interpreting the most commonly used sensory evoked potentials. They do not consider new techniques that are becoming available or techniques that are only used occasionally. In particular, they do not consider the use of sensory evoked potentials in the operating room. ${ }^{\text {? }}$

\section{Clinical Interpretation of Evoked Potentials}

The physician interpreting the evoked potentials must fulfill the criteria for membership in the Canadian Society of Clinical Neurophysiologists. The physician should have either a fellowship from the Royal College of Physicians and Surgeons or a Ph.D. in a basic science discipline related to clinical neurophysiology, and at least 6 months of full-time supervised training in electroencephalography or electromyography in a laboratory that performs at least 5 clinical evoked potential studies per week. Training requirements could, of necessity, be waived for experienced neurophysiologists who learned their evoked potential skills before formal training was available.

\section{Evoked Potential Technologists}

The Canadian Board of Registration of Electroneurophysiology Technologists examines the competence of technologists performing EEGs. The Canadian Association of Clinical Neurophysiologists examines the competence of technologists performing EMGs. The American Board of Registered EEG Technologists provides specialized examinations in the techniques for recording the evoked potentials. An evoked potential technologist should be accredited by one of these examining boards.

\section{General Technical Requirements}

1) The equipment must have leakage currents and ground resistances that are acceptable to the Canadian Standards Association.

2) The evoked potentials should be recorded using cup or disc electrodes. The skin should be gently abraded so that the inter-electrode impedances are below $5000 \mathrm{Ohms}$.

3) The differential amplifiers should have an input impedance of at least $10 \mathrm{MOhms}$ and a common mode rejection ratio of at least $80 \mathrm{~dB}$. The noise level of the amplifiers should be less than $2 \mu \mathrm{V}$ rms over the frequency range $1-5000 \mathrm{~Hz}$ when both inputs are connected to ground.

4) The equipment should have at least 4 channels of amplification available for simultaneous recordings.

5) The averaging equipment should provide A/D conversion at rates sufficient to provide at least 256 points per channel per averaging sweep.

6) The equipment should allow artifact-contaminated trials to be rejected automatically from the averaging process.

7) The equipment must provide some means of assessing the signal-to-noise ratios for all recordings. The easiest way to evaluate this is to allow the superimposition of replicate tracings.

8) There should be some means of permanently storing the recorded waveforms on paper and/or on some electronic storage medium.

\section{Normative Data}

It is essential that all laboratories run a group of at least 10 normal adult subjects under the usual recording conditions for the evoked potentials being evaluated. The data obtained from this group of normal subjects should be 
compared to normative data available in the literature. If there are no significant differences in either the mean values or the standard deviations, the laboratory can use the published literature to provide age- and gender-specific normative data. If the laboratory will be testing children, it should evaluate a group of normal subjects at an age equivalent to the youngest subjects that will be tested (e.g., neonates) and another group at an age near the middle of the age-range of children tested. These evaluations will ensure that the laboratory is recording and measuring data in the same way as the studies that will be used for normative data. During infancy and childhood the evoked potentials change rapidly with age. In infancy significant changes in the measurements occur monthly. Sufficient data must therefore be available to provide age- and gender-matched normative values for the patients being tested.

The limits of normal are usually expressed in terms of a value beyond which measurements in only 1 in 100 normal subjects will occur. In evaluating latencies one only accepts as abnormal latencies that are long. Since these values are approximately normal in their distribution one can consider the upper limits of normal to be 2.3 standard deviations above the mean. Since amplitude-measurements are not usually normally distributed it is not possible to assess the $1 \%$ limits using standard deviations and formal tolerance limits must be estimated.

\section{Visual Evoked Potentials}

The most commonly used stimulus is a reversing black and white checkerboard. The contrast between the black and white squares of the stimulus is evaluated by dividing the difference between the brightness of the white squares and the brightness of the black squares by the sum of these two values. This contrast should be at least $50 \%$. The brightness of the white squares should be at least $100 \mathrm{~cd} / \mathrm{m}^{2}$. The time for the change between the two checkerboards stimuli should be less than $20 \mathrm{~ms}$. Each check should subtend about $0.5^{\circ}$ of visual arc and the entire stimulus should subtend an angle of greater than $5^{\circ}$. The rate of reversal should be between $1-2 /$ second. The stimulus should be viewed monocularly. The patient should wear glasses to correct for any refractive error. The patient should be observed during the recording to ensure that he or she is fixating on the centre of the stimulus.

Flashes may be used as stimuli when the patient cannot fixate on or see the checkerboard stimulus, e.g., infants or patients with cataracts. Normative data for flash-evoked responses are more variable than for the pattern-reversal responses. Furthermore, since the responses are highly specific to the exact type of flash, it is difficult to use published norms.

The visual evoked potentials are recorded from the midoccipital region relative to the mid-frontal region. The filter band-pass of the amplifier should be $1-100 \mathrm{~Hz}$. The response should be recorded using a sweep of at least $250 \mathrm{~ms}$. Averaging should be carried out over 100-200 trials.
The major peak of the pattern-reversal response is an occiput-positive peak at approximately $100 \mathrm{~ms}$. Recordings from the inion and from right and left occipital regions may be helpful in identifying the response. Furthermore, they may provide some evaluation of its symmetry.

\section{Auditory EvoKed Potentials}

The stimulus should be a click obtained by passing a 100 $\mu$ square wave through a standard audiometric earphone. For neurological purposes, the polarity of the initial deflection of the click should result in a rarefaction pulse at the eardrum. The intensity of the stimulus should be between $60-90 \mathrm{~dB}$ above normal adult thresholds for this stimulus (nHL). For neurological purposes, clicks should be presented monaurally at rates between 10 and $30 / \mathrm{s}$.

The responses are recorded between an electrode at the vertex or mid-frontal region and one at the earlobe or mastoid of the ear being stimulated. The filter band-pass of the amplifier should be $30-3000 \mathrm{~Hz}$. The response should be recorded over a sweep between 10-15 ms. Between 1000 and 4000 trials will be needed for averaging.

The auditory brainstem response contains between 5 and 7 vertex-positive waves of which three are sufficiently reliable for clinical measurement: I, III, and V. For neurological purposes the I-III and I-V interpeak latencies are the most important measurements. In many patients it may be necessary to record the response at different intensities and at different rates in order to identify the components more clearly. An electrode in the external auditory meatus may assist in the identification of wave $\mathrm{I}$.

\section{Somatosensory Evoked Potentials}

The recommended stimulus is a constant-current pulse supplied through electrodes located on the skin over the nerves being evaluated. The point of stimulation is close to the cathode. The duration is usually between 0.1 and $0.3 \mathrm{~ms}$. Stimuli are presented at rates near $5 / \mathrm{s}$. Faster rates are usually uncomfortable. The two most commonly used nerves are the median nerve at wrist and the posterior tibial nerve at the ankle. The intensity of the stimulus is adjusted to a level that is $10-20 \%$ higher than the threshold for eliciting a visible motor twitch.

The somatosensory responses are recorded using a filter bandpass of $10-3000 \mathrm{~Hz}$ although the low-frequency cutoff may be as high as $30 \mathrm{~Hz}$ if the cutoff slope is not more than 6 $\mathrm{dB} /$ octave. Between 500 and 2000 trials are usually averaged together. The sweep duration is $40-50 \mathrm{~ms}$ for the median nerve response and $60-80 \mathrm{~ms}$ for the posterior tibial nerve response.

For the median nerve response, recordings are taken from 3 locations: the brachial plexus, the spinal cord and the cortex. Brachial plexus responses can be recorded from an electrode on Erb's point or the adjacent clavicle using a reference on the contralateral shoulder. The spinal cord responses are 
recorded from the posterior neck at the level of the $\mathrm{C} 5$ cervical vertebra using a reference on the mid-frontal scalp or a non-cephalic reference (contralateral shoulder or hand). The cortical response should be recorded from a location on the scalp contralateral to the stimulation mid-way between $\mathrm{C} 3$ or $\mathrm{C} 4$ and $\mathrm{P} 3$ or $\mathrm{P} 4$. This recording can be referenced to the contralateral cortex, the mid-frontal region or the contralateral mastoid. A recording from the ipsilateral hemisphere can help to distinguish cortical and brainstem responses.

The major components to be recognized are 3 negative waves: the brachial plexus wave peaking at approximately 10 $\mathrm{ms}$, the cervical cord response at approximately $14 \mathrm{~ms}$ and the initial cortical response at approximately $20 \mathrm{~ms}$.

For the posterior tibial nerve response, a recording should be obtained from the lumbar spinal cord with an electrode over the lower back at the L2 vertebra referred to the hip or to the sternum. If the lumbar response is not clearly recognizable, the nerve action potential of the posterior tibial nerve at the knee can be recorded to demonstrate normal (or abnormal) function in the nerve. The cortical response is recorded maximally from an electrode mid-way between the vertex and the mid-parietal location. This recording can be referred to a mid-frontal electrode. The recognition of the cortical response can be facilitated by making additional recordings from the left and right parietal regions to demonstrate the specific scalp distribution of the response. These sites should be sampled before concluding that a response is absent or abnormal.

The major components to be recognized in the response to posterior tibial nerve stimulation are the lumbar negativity at approximately $20 \mathrm{~ms}$ and the initial cortical positive wave at approximately $39 \mathrm{~ms}$. The latencies of both these components will vary with the height of the subject.

\section{SENSORY Evaluation}

It is essential that a minimum sensory examination be obtained at the same time as the evoked potentials. This would include visual acuity testing for the visual evoked potentials, click thresholds for auditory potentials, and the threshold for the electrical stimulus for somatosensory evoked potentials. In the case of abnormal evoked potentials, the clinical interpretation of the results will be facilitated by a full pure-tone audiogram, visual-field testing and the results of somatosensory examination using pin, light touch, vibration and position.

\section{Evoked Potential Reports}

The evoked potential report should include the following information:

(i) Laboratory identification.

(ii) Patient identification.

(iii) The object of the investigation.

(iv) A description of the evoked potential measurements. These measurements should include the latencies of the identified peaks.

(v) Interpretation.

The responses should be interpreted as normal or as abnormal. If abnormal, the findings should be interpreted in terms of the underlying pathophysiology. This may be then related to possible clinical conditions, with the provisos that an evoked potential abnormality may be able to indicate the location of some dysfunction but not its specific pathology, and that these findings are only part of the overall clinical diagnosis.

\section{REFERENCES}

1. Chiappa K. Evoked Potentials in Clinical Medicine. Second Edition. New York: Raven Press, 1989.

2. Halliday AM. Evoked Potentials in Clinical Testing. Second Edition. Edinburgh: Churchill Livingstone, 1993.

3. American Electroencephalographic Society. Guidelines in EEG and Evoked Potentials. J Clin Neurophysiol 1986; 3 (Suppl 1): 1-152.

4. American Electroencephalographic Society. Guidelines for Evoked Potentials. May, 1992. (These revised guidelines are available from the American EEG Society and will be published in J Clin Neurophysiol).

5. American Electroencephalographic Society. Guidelines for Writing Clinical Evoked Potential Reports. J Clin Neurophysiol 1991; 8 : 85-87.

6. Halliday AM. Standards of clinical practice for the recording of evoked potentials (EPs). In: International Federation of Societies for Electroencephalography and Clinical Neurophysiology, Recommendations for the Practice of Clinical Neurophysiology. Amsterdam: Elsevier, 1983; 69-80.

7. American Electroencephalographic Society. Guidelines for Intraoperative Monitoring of Sensory Evoked Potentials. J Clin Neurophysiol 1987; 4: 397-416. 